OPEN ACCESS

Edited by:

David K. Mills,

Louisiana Tech University,

United States

Reviewed by:

Andre D. R. Silva,

Brazilian Air Force Academy, Brazil

Chunguang Yang,

Institute of Metals Research (CAS),

China

*Correspondence:

Peter Homolka

peter.homolka@meduniwien.ac.at

Specialty section:

This article was submitted to Biomaterials,

a section of the journal

Frontiers in Bioengineering and

Biotechnology

Received: 08 September 2021

Accepted: 25 October 2021

Published: 29 November 2021

Citation:

Ma X, Buschmann M, Unger E and Homolka P (2021) Classification of $X$ Ray Attenuation Properties of Additive Manufacturing and 3D Printing Materials Using Computed Tomography From 70 to $140 \mathrm{kVp}$. Front. Bioeng. Biotechnol. 9:763960.

doi: 10.3389/fbioe.2021.763960

\section{Classification of X-Ray Attenuation Properties of Additive Manufacturing and 3D Printing Materials Using Computed Tomography From 70 to $140 \mathrm{kVp}$}

\author{
Xiangjie Ma ${ }^{1}$, Martin Buschmann ${ }^{2}$, Ewald Unger ${ }^{1}$ and Peter Homolka ${ }^{1 *}$ \\ ${ }^{1}$ Center for Medical Physics and Biomedical Engineering, Medical University of Vienna, Vienna, Austria, ${ }^{2}$ Division of Medical \\ Radiation Physics, Department of Radiation Oncology, Medical University of Vienna, Vienna, Austria
}

Additive manufacturing and 3D printing is particularly useful in the production of phantoms for medical imaging applications including determination and optimization of (diagnostic) image quality and dosimetry. Additive manufacturing allows the leap from simple slab and stylized to (pseudo)-anthropomorphic phantoms. This necessitates the use of materials with $\mathrm{x}$-ray attenuation as close as possible to that of the tissues or organs mimicked. X-ray attenuation properties including their energy dependence were determined for 35 printing materials comprising photocured resins and thermoplastic polymers. Prior to measuring $\mathrm{x}$-ray attenuation in CT from 70 to $140 \mathrm{kVp}$, printing parameters were thoroughly optimized to ensure maximum density avoiding too low attenuation due to microscopic or macroscopic voids. These optimized parameters are made available. CT scanning was performed in a water filled phantom to guarantee defined scan conditions and accurate HU value determination. The spectrum of $\mathrm{HU}$ values covered by polymers printed using fused deposition modeling reached from -258 to $+1,063$ at $120 \mathrm{kVp}(-197$ to $+1,804$ at $70 \mathrm{kVp}$, to -266 to +985 at $140 \mathrm{kVp}$, respectively). Photocured resins covered 43 to $175 \mathrm{HU}$ at $120 \mathrm{kVp}(16-156$ at 70 , and $57-178$ at $140 \mathrm{kVp})$. At $120 \mathrm{kVp}$, ASA mimics water almost perfectly (+2 HU). HIPS (-40 $\mathrm{HU}$ ) is found close to adipose tissue. In all photocurable resins, and 17 printing filaments $\mathrm{HU}$ values decreased with increasing beam hardness contrary to soft tissues except adipose tissue making it difficult to mimic water or average soft tissue in phantoms correctly over a range of energies with one single printing material. Filled filaments provided both, the HU range, and an appropriate energy dependence mimicking bone tissues. A filled material with almost constant $\mathrm{HU}$ values was identified potentially allowing mimicking soft tissues by reducing density using controlled under-filling. The measurements performed in this study can be used to design phantoms with a wide range of $\mathrm{x}$-ray contrasts, and energy dependence of these contrasts by combining appropriate materials. Data provided on the energy dependence can also be used to correct contrast or contrast to noise ratios from phantom measurements to real tissue contrasts or CNRs.

Keywords: additive manufacturing, 3D printing, computed tomography, quality control, radiographic phantoms, $\mathbf{x}$-ray, phantom materials 


\section{INTRODUCTION}

Due to their great potential, additive manufacturing and 3D printing have become indispensable technologies in medicine as a whole, in medical imaging in particular. This is not only due to the increasing number of developers and scientists eagerly embracing the new method for realizing their projects, but also by the rapid technological development of $3 \mathrm{D}$ printing processes and materials available to the imaging community. Based on the number of publications abstracted in the Web of Science (Clarivate Analytics LLC, Philadelphia, United States) in the last 3 years (2019 to August 2021), Radiology, Nuclear Medicine and Medical Imaging ranked just behind Multidisciplinary Material Science (\#1), Biomedical Engineering (\#2), Biomaterials (\#3), Surgery (\#4), and Dentistry (\#5), when purely technical fields just applying medical imaging and related methods were omitted. These omissions include applied physics and engineering manufacturing research, where additive manufacturing and medical/dental appliances were used. The increasing importance of additive manufacturing and $3 \mathrm{D}$ printing in medical and dental applications as a whole, and in medical imaging in particular, is demonstrated best not only by the total number of indexed scientific publications, but also by their relative share, where a linear rise can be seen in the last decade. For both, 3D printing and additive manufacturing in medical and dental applications, and in medical imaging, the relative share of all indexed scientific publications increased by over $40 \%$ in 3 years (from 2017 to 2020).

In medical imaging, the most important fields where $3 \mathrm{D}$ printing and additive manufacturing are applied include design and production of phantoms for all kinds of modalities including CT, US, nuclear medicine modalities like PET and SPECT, projective imaging and mammography (Filippou and Tsoumpas, 2018). In 2D and 3D X-Ray imaging and dosimetry, $3 \mathrm{D}$ printing and additive manufacturing paved the way from rather simple phantoms, like stylized phantoms (Smet et al., 2018) to almost anthropomorphic phantoms for projective imaging (Irnstorfer et al., 2019), mammography (Carton et al., 2011; Kiarashi et al., 2015; Schopphoven et al., 2019) or dosimetry (Homolka et al., 2017). While simple test objects and phantoms are only capable of assessing technical image quality, advanced phantoms allow progressing towards determination and optimization of task specific diagnostic image quality. Anthropomorphic three dimensional phantoms capable of producing (nearly) realistic tissue background patterns-called "anatomic noise"- in which lesions can be embedded represent a milestone in performing investigations of lesion detectability, optimizing procedure settings and system evaluation (Ivanov et al., 2018). 3D printing has the potential to bring medical physicists developing these phantoms closer to being able to produce these structured anthropomorphic backgrounds (Solomon et al., 2014). On the other hand, printing realistic anthropomorphic lesions with appropriate materials and extremely fine spatial resolutions making them suitable even for mammography and tomosynthesis applications has been shown to be feasible, even if the anatomic background is provided by a "semi-anthropomorphic" model composed of acrylic spheres of various sizes (Cockmartin et al., 2017), or PVC film submerged in a paraffin gel with a non-uniform distribution (Sousa et al., 2018).

However, in medical imaging applications, printing materials are usually selected based on their properties used by the imaging modality to create image signals and contrasts, rather than the properties for which they are typically designed for. Development of these materials is mostly driven by industrial additive manufacturing applications. In medical imaging applications, however, totally different properties are important. These include proton density and relaxation time constants in MRI, acoustic properties and echogenicity in ultrasound imaging, and $\mathrm{x}$-ray attenuation properties in $\mathrm{CT}$ and projective $\mathrm{x}$-ray imaging.

Still, and sometimes against the claims in publications, most existing "anthropomorphic phantoms" should rather be viewed as being "semi-" or "pseudo-anthropomorphic". In order to be really anthropomorphic, a phantom would not only represent the anatomy with the spatial resolution of the phantom at least resembling the intrinsic spatial resolution of the imaging modality, but also would the materials used to represent tissues need to imitate the interaction properties over the full range of acquisition settings of the modality or modalities they are intended for. Limiting the scope to $\mathrm{x}$-ray imaging (projective imaging like general radiography, fluoroscopy and interventional radiology, and computed tomography), this would necessitate that $x$-ray attenuation was very closely equal to the attenuation of the tissue to be imitated over the full range of X-ray photon energies present in the polychromatic beam, from the minimum to maximum $\mathrm{kVp}$ settings used. However, this requirement is usually relaxed by demanding that the total attenuation and the energy dependence of the attenuation shall resemble the energy dependence of the materials or tissues mimicked as closely as possible. However, this is more easily achieved for projective imaging, where in most cases it is sufficient to imitate the mass attenuation coefficient and possibly add air gaps as was done in the simple slab phantoms used for dosimetry and dose audits, like the NEXT and CDRH phantoms (Suleiman et al., 1999; Spelic et al., 2004; IAEA, 2007), than for computed tomography where the linear attenuation coefficient needs to imitated (Homolka and Nowotny, 2002). The latter usually results in the necessity of lowering the mass density if soft tissues or water are imitated by adding filling materials with very low density if curable liquid resins were used (White et al., 1977), or using low density thermoplastic polymers or a mixture of these (Kalender et al., 1988; Homolka and Nowotny, 2002). In castable resin based materials mimicking soft tissue or water, typically hollow air filled phenolic microspheres were used (White et al., 1977).

In the underlaying physics the energy dependence of the $\mathrm{x}$-ray mass attenuation coefficient being equal to the tissue that is mimicked, translates to the ratio of the contribution of inelastic scattering to the photoelectric effect (absorption) being as similar as possible. In contrast to therapeutic photon energies this is in the lower keV range especially complicated by the contribution of the photoelectric effect. However, this can usually be only satisfied for a limited photon energy range, that must include all $\mathrm{kVp}$ and filtration settings possibly used. In mammography 
this includes also all anode filter combinations. Using thermoplastic and resin-based polymers, imitating the x-ray attenuation of soft tissues is especially difficult for photon energies below $30 \mathrm{keV}$ due to the low effective atomic number of the polymers (Homolka et al., 2002). Therefore, phantom materials need to be formulated (if possible) or selected (in case of $3 \mathrm{D}$ printing) carefully for the application and photon energy range used. Materials simulating a given tissue with respect to $\mathrm{x}$-ray attenuation at general radiography photon energies will most likely not be suitable at mammography energies, and vice versa. In this work the main focus is characterizing available $3 \mathrm{D}$ printing materials with regard to their $\mathrm{x}$-ray attenuation for tungsten spectra from 70 to $150 \mathrm{kVp}$ covering typical photon energies used in general radiography and computed tomography. It needs to be stressed, that in phantoms used for optimizing procedure parameters like beam hardness $(\mathrm{kVp}$ and/or filtration, e.g.) it is imperative that the energy dependence of the phantom-and thus, the energy dependence of any important radiographic contrast, SNR or CNR determined-is correct. The same holds true for phantoms used in dosimetry or dose determination, especially if energy dependent dosemeters like TLDs or semiconductor dosemeters are employed, or wide polychromatic spectra are used.

Characterization and also reproducibility of $\mathrm{x}$-ray attenuation properties of additive manufacturing materials is complicated by various issues. Exact chemical composition is often not available to the end user, and it may change in different batches since materials are usually developed further to improve printing results or simplify the printing process. Another issue is found with materials, where printing parameters influence the final mass density of the printout. This needs to be addressed properly, otherwise a too low print density due to a suboptimal parameter setting would be incorrectly attributed to the material and not to the process parameters. This is particularly important for printing processes in which the polymers are melted in air during the printing process, such as FDM (Fused Deposition Modeling) or SLS (Selective Laser Sintering).

The objective of this work was to find and describe optimum printing parameters for a wide range of additive manufacturing materials, and to quantify their $x$-ray attenuation properties as exactly as possible using CT scans from the lowest to the highest $\mathrm{kVp}$ value currently available in our institution.

\section{MATERIALS AND METHODS}

\section{Printing Materials and Technologies Used}

Thermoplastic polymers available for fused deposition modeling (FDM) printers were printed on an Ultimaker $2+$ with standard $0.4 \mathrm{~mm}$ nozzle diameter (Ultimaker BV, Utrecht, Netherlands). A wide range of polymer filaments was selected with the scope to include a representative cross section of unfilled base polymers. Filled polymers include filaments with potentially useful $\mathrm{x}$-ray attenuation properties including materials filled with mineral and metal powders potentially allowing mimicking hard tissues in radiographic phantoms.

UV curable resin samples were printed with polyjet and stereolithography (SLA) printers. A Stratasys Connex 3 Objet 500 polyjet printer (Stratasys Ltd., Eden Prairie, United States), a Formlabs Form 2 (Formlabs Inc., Somerville, United States, SLA) and an Anycubic Photon open resin printer (Shenzhen Anycubic Technology Co., Ltd., Shenzhen, China, SLA) were employed. Materials printed with these technologies were chosen to include both, rigid and flexible resins, and represent a cross section of readily available resins from different manufacturers. Ceramic materials were excluded (as also were heavily metal filled FDM filaments, like such filled with metals with higher atomic number than aluminum) since their typically high $\mathrm{x}$-ray attenuation does not allow quantitative measurements in CT due to severe beam hardening and quantum starvation artifacts.

Printing materials and printers used for the respective materials are summarized in Tables 1 (FDM printer) and 2 (Polyjet and SLA printers).

The polyjet technology does not allow manual settings of print parameters. The same holds true for SLA samples printed with one of the printers (Formlabs Form 2), since printing materials are recognized by the printer, and parameters set automatically. On the Anycubic Photon SLA printer, print settings recommended by the resin manufacturers were used after verification of their appropriateness from test object printouts.

\section{Optimization of Printing Parameters in FDM}

In FDM general purpose printing, the density of the printed objects is usually below the density of the filament used. Inherent to the printing process, and dependent on the layer thickness and the viscosity of the melted polymer, the deposition of material results in microscopic voids between adjacent polymer deposition lines. In case this effect is strongly present, the layer structure can be seen on the outer surface, and transparent materials appear opaque. However, rough surfaces can also be caused by too strong over-extrusion, so printing parameters are usually optimized for smooth outer surfaces. Unless controlled under-extrusion is used in a reproducible fashion to reduce density and thus $\mathrm{x}$-ray attenuation in radiographic phantoms, it is important to ensure maximum filling ratios, i.e., maximum packing, in the samples used to measure and characterize attenuation properties of printing materials. Optimally, the measured density of the printed samples resembles the filament density.

In this work, starting from printing parameters optimized for general purpose prints, printing parameters were fine tuned to result in the maximum filling ratio, i.e., maximum achievable mass density. Provided the specification of filament density was available and correct, the aim of the parameter optimization was to produce samples with a mass density identical to the filament density. In a first step printing parameters like nozzle temperature and printing speed were optimized using the \#3DBenchy test object (\#3DBenchy, 2021) available as STL file under the Creative Commons License. The aim of this first step was to achieve optimum printing results including minimal artifacts like stringing, warping, surface roughness, an even surface structure, and dimensional accuracy. In the next step 
TABLE 1 | Polymer filaments used on the FDM printer.

Filament name

Manufacturer

\section{Manufacturer}

Code/EAN

High attenuation filaments

$\begin{array}{llll}\text { Vinyl } & \text { Polyvinyl chloride } & \text { Vinyl } 303 \text { natural } & \begin{array}{l}\text { Fillamentum Manufacturing Czech s.r.o., } \\ \text { Hulín, Czech Republic }\end{array} \\ \text { PLA/stone } & \text { PLA filled with 50\% powdered stone } & \text { StoneFil Pottery Clay } & \text { Formfutura BV, Nijmegen, Netherlands } \\ \text { PLA/chalk } & \text { PLA with chalk powder } & \text { PLA Mineral natural } & \text { Fiberlogy SA, Brzezie, Poland } \\ \text { 285STONEFIL- }\end{array}$

Filled filaments with medium and low attenuation

\begin{tabular}{|c|c|c|c|c|}
\hline $\begin{array}{l}\text { PLA-PHA } \\
\text { Glow }\end{array}$ & PLA/PHA filled with phosphorescent pigment & GlowFill & colorFabb BV, Belfeld, Netherlands & 8719033555136 \\
\hline $\begin{array}{l}\text { PLA-PBAT } \\
\text { bio carbon }\end{array}$ & PLA/PBAT based biocompound with carbon fibers & $\begin{array}{l}\text { GreenTEC PRO } \\
\text { Carbon }\end{array}$ & Extrudr FD3D GmbH, Lauterach, Austria & 9010241426973 \\
\hline PLA/Al & PLA filled with $10 \%$ aluminum powder & $\begin{array}{l}\text { Aptofun Metal Filament } \\
\text { Aluminium }\end{array}$ & Aptotec UG, Tübingen, Germany & B01ITNXRWD \\
\hline PLA/wood & PLA filled with $40 \%$ grinded wood particles & EasyWood Birch & Formfutura & $\begin{array}{l}\text { 285EWOOD-BIRCH- } \\
0500\end{array}$ \\
\hline $\begin{array}{l}\text { PLA-PHAV } \\
\text { Cork }\end{array}$ & $\begin{array}{l}\text { PLA/PHA (Polylactic acid/Polyhydroxyalkanoate) blend } \\
\text { filled with cork powder }\end{array}$ & corkFill & colorFabb & 8719033555327 \\
\hline $\begin{array}{l}\text { PETG mod./ } \\
\text { Carbon }\end{array}$ & $\begin{array}{l}\text { HDglass (PETG based polymer blend) with } 20 \% \text { carbon } \\
\text { fibers }\end{array}$ & CarbonFil & Formfutura & $\begin{array}{l}\text { 175CARBFIL-BLCK- } \\
0500\end{array}$ \\
\hline
\end{tabular}

PLA and PLA/PBAT based filaments

\begin{tabular}{|c|c|c|c|c|}
\hline PLA & Polylactic acid & PLA transparent & Ultimaker BV, Utrecht, Netherlands & 1614 \\
\hline PLA 2 & Polylactic acid & PLA Sparkly Silver & $\begin{array}{l}\text { Shenzhen Eryone Technology Co. Ltd., } \\
\text { Shenzhen, China }\end{array}$ & $\begin{array}{l}\text { GPLA-SILVER-175- } \\
1000\end{array}$ \\
\hline $\begin{array}{l}\text { PLA } \\
\text { PBAT bio }\end{array}$ & $\begin{array}{l}\text { PLA/PBAT (polylactic acid/polybutylene adipate } \\
\text { terephthalate) based biocompound }\end{array}$ & GreenTEC PRO natural & Extrudr & 9010241426034 \\
\hline
\end{tabular}

PET and PETG based filaments

\begin{tabular}{llll} 
PET & Polyethylene terephthalate & EPR InnoPET & \multicolumn{1}{c}{ Innofil3d BV, Emmen, Netherlands } \\
PET mod. & PET copolyesther, Eastman Amphora 3D Polymer & XT-Clear & colorFabb \\
& AM1800 & n719033553019 \\
PET mod. 2 & $\begin{array}{l}\text { PET copolyesther, Eastman Amphora 3D Polymer } \\
\text { AM3300 }\end{array}$ & nGen clear & colorFabb \\
PETG mod. & PETG based polymer blend & HDglass clear & Formfutura
\end{tabular}

ABS, ASA and ASA based filaments

$\begin{array}{llll}\text { ABS } & \text { Acrylonitrile butadiene styrene } & \text { ABS transparent } & \text { Verbatim } \\ \text { ASA } & \text { Acrylonitrile styrene acrylate } & \text { ASA Extrafill natural } & \text { Fillamentum } \\ \text { ASA mod. } & \text { Modified ASA } & \text { ApolloX White } & \text { Formfutura }\end{array}$

PS, PP, PC and Polyamid based filaments

$\begin{array}{llll}\text { HIPS } & \text { High impact polystyrene } & \text { HIPS wonderous white ICE Filaments, Ham, Belgium } & \text { ICEFIL3HPS170 } \\ \text { PP } & \text { Polypropylene } & \text { PP transparent } & \text { Verbatim GmbH, Eschborn, Germany } \\ \text { PP light } & \begin{array}{l}\text { Polypropylene with 25\% hollow borosilicate glass } \\ \text { microspheres }\end{array} & \text { Pegasus PP Ultralight } & \text { Formfutura } \\ \text { PC } & \text { Polycarbonate } & \text { PC-Plus transparent } & \text { Polymaker, Shanghai, China } \\ \text { Nylon } & \text { Polyamide } & \text { Nylon transparent } & \text { Ultimaker }\end{array}$

PU based filaments

TPU Thermoplastic polyurethane TPU transparent $\quad 9010241152001$

Notes: Manufacturer details are only shown on first appearance. In case no manufacturer code was available, EAN is stated for identification. 
TABLE 2 | Polyjet and SLA resins used.

\begin{tabular}{|c|c|c|c|c|}
\hline & Printer used & Resin name & Manufacturer & Manufacturer code \\
\hline \multicolumn{5}{|c|}{ Rigid SLA resins } \\
\hline Vero white & $\begin{array}{l}\text { Stratasys } \\
\text { Objet } 500\end{array}$ & Vero Pure White & Stratasys Ltd., Eden Prairie, United States & OBJ-03327 \\
\hline Vero clear & $\begin{array}{l}\text { Stratasys } \\
\text { Objet } 500\end{array}$ & Vero Clear & Stratasys & OBJ-03271 \\
\hline Vero blue & $\begin{array}{l}\text { Stratasys } \\
\text { Objet } 500\end{array}$ & Vero Blue & Stratasys & OBJ-03204 \\
\hline FL clear & Formlabs Form 2 & Formlabs Clear Resin & Formlabs Inc., Somerville, United States & RS-F2-GPCL-04 \\
\hline PCre clear & Anycubic Photon & Prima Creator Value UV/DLP resin clear & Prima Printer Nordic AB, Malmö, Sweden & PV-RESIN-B405-CL \\
\hline $\begin{array}{l}\text { AC trans- } \\
\text { lucent }\end{array}$ & Anycubic Photon & $\begin{array}{l}\text { Anycubic 3D Printing UV Sensitive Resin Basic, } \\
\text { Translucent green }\end{array}$ & $\begin{array}{l}\text { Shenzhen Anycubic Technology Co., Ltd., } \\
\text { Shenzhen, China }\end{array}$ & AB-POT048 \\
\hline \multicolumn{5}{|c|}{ Flexible SLA resins } \\
\hline Tango & $\begin{array}{l}\text { Stratasys } \\
\text { Objet } 500\end{array}$ & Tango Plus (translucent) & Stratasys & OBJ-03224 \\
\hline FL flex & Formlabs Form 2 & Formlabs Flexible Resin & Formlabs & RS-F2-FLGR-02 \\
\hline FL elastic & Formlabs Form 2 & Formlabs Elastic Resin & Formlabs & RS-F2-ELCL-01 \\
\hline PCen flex & Anycubic Photon & PhotoCentric3D UV LCD Resin Flexible clear & Photocentric Ltd., Peterborough, United Kingdom & PHODCL01UVFLEX \\
\hline
\end{tabular}
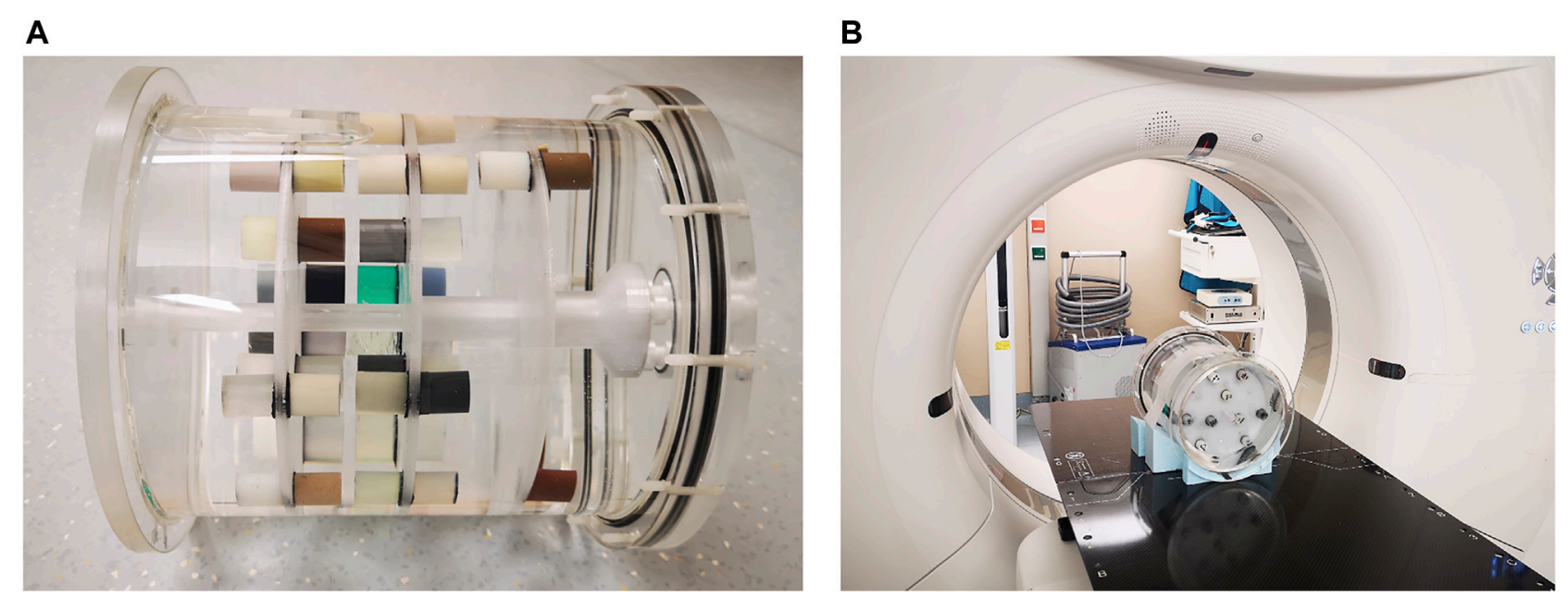

FIGURE 1 | Printed sample cylinders mounted into a water filled phantom for CT scanning.

homogeneous cylinders with $15 \mathrm{~mm}$ diameter and $20 \mathrm{~mm}$ height were printed and their mass density determined gravimetrically. In case density was lower than filament density, over-extrusion was systematically applied by increasing the flow rate. If increasing flow rate up to a point where over-extrusion artifacts became visible or dimensional accuracy decreased did not result in the desired density, layer height was decreased until either the specified mass density was achieved, or until further increase of over extrusion and reduction of layer height did not result in increased density. Layer highs available on the printer used were $0.15,0.10$, and $0.6 \mathrm{~mm}$, respectively.

\section{Determination of CT Values}

The printed cylinders were mounted in a water filled phantom for scanning (Figure 1) to ensure Hounsfield number accuracy avoiding systematic HU number errors resulting from cupping (or reverse cupping) artifacts. The cylinders were distributed on six levels each consisting of an inner and an outer circle. The inner circle accommodated a maximum of three cylinders, the outer eight. Since the total number of cylinders scanned was considerably smaller than available positions in the phantom, the cylinders were distributed leaving unfilled positions minimizing beam hardening effects. Scanning was performed at $70,80,100,120$ and $140 \mathrm{kVp}$ applying a modified head protocol with 32 times $0.6 \mathrm{~mm}$ total collimation, and a medium soft tissue kernel (H40s). The rotation time was set to $1 \mathrm{~s}$ and a pitch factor of 0.55 was selected to allow higher effective $\mathrm{mAs}$ resulting in reduced image noise compared to clinical protocols. $\mathrm{mAs}$ were set to their respective maximum values for $70 \mathrm{kVp}$ (900 mAs) and $80 \mathrm{kVp}(1,100 \mathrm{mAs})$ resulting 


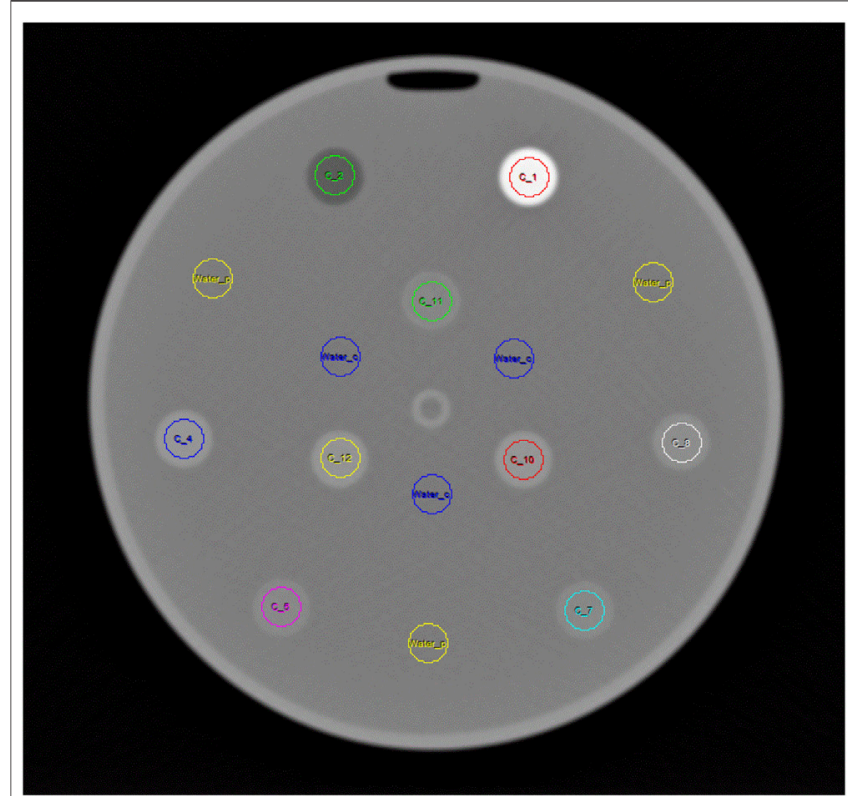

FIGURE 2 | Sample CT slice showing ROIs used for $\mathrm{HU}$ measurement in the printed cylinders arranged in two circles. Additional ROls used to measure $\mathrm{HU}$ in the water are shown in blue (inner circle) and yellow (outer circle).

in a $\mathrm{CTDI}_{\mathrm{vol}}$ of 31 and $60 \mathrm{mGy}$, respectively. For $100-140 \mathrm{kV} \mathrm{mAs}$ were set to result in a CTDI of $100 \mathrm{mGy}( \pm 2 \mathrm{mGy})$. Slices were reconstructed with $1 \mathrm{~mm}$ slice thickness and increment.

CT images were evaluated with Analyze 12.0, Biomedical Image Resource (Mayo Clinic, Rochester, United States). To avoid HU number inaccuracies from partial volume effects, the outermost slices for each cylinder were excluded from the analysis, and the respective ROIs were smaller $(10 \mathrm{~mm})$ compared to the actual diameter of the cylinders of $15 \mathrm{~mm}$ (Figure 2). The evaluated height of every $20 \mathrm{~mm}$ high cylinder comprised 17 adjacent $1 \mathrm{~mm}$ thick slices avoiding top/bottom slices for the same reason. HU values measured for the printed samples were corrected by subtracting the actually measured Hounsfield value in water in the corresponding circle to compensate for eventual cupping and beam hardening not perfectly corrected by the scanner's beam hardening correction. Therefore, three additional ROIs each were placed in the water filled background at the diameter of the inner, and the outer circle, respectively (Figure 2).

\section{RESULTS}

\section{Optimization of Printing Parameters for FDM}

Printing parameters resulting in optimum print quality, dimensional accuracy and the maximum achievable mass density are summarized in Table $\mathbf{3}$ for all 25 thermoplastic filaments printed using fused deposition modeling. Five thermoplastic polymer filaments could be printed with a mass density exactly resembling the filament density $\left( \pm 0.00 \mathrm{~g} / \mathrm{cm}^{3}\right)$ indicating perfectly solid printouts. These included vinyl, polylactic acid and polycarbonate, as well as filled materials based on PLA/PHA and modified PETG. Eleven materials printed with a deviation of $0.01 \mathrm{~g} / \mathrm{cm}^{3}$ indicating near optimum achievable density. These materials included all tested PET and modified PET/PETG materials, ABS, ASA and polypropylene. Nine of those exhibited a density $0.01 \mathrm{~g} / \mathrm{cm}^{3}$ lower than specified filament density, while two (a wood filled PLA, and TPU) exhibited a density $0.01 \mathrm{~g} / \mathrm{cm}^{3}$ higher than specified.

One carbon fiber filled biopolymer exhibited a density $17.39 \%$ higher than specified, while the same unfilled base filament had a density $2.88 \%$ below its specification. However, according to the manufacturer, the specified density for the carbon fiber filled polymer was based on calculations rather than measurements. A cork filled PLA/PHA filament exceeded the specified density by $2.54 \%$, a modified ASA filament by $1.8 \%$. A polypropylene filament filled with glass microspheres used to lower the density exhibited an even $2.67 \%$ too low density, and Nylon and HIPS could only be printed with a density $0.02 \mathrm{~g} / \mathrm{cm}^{3}$ lower than the specified filament density. A further increase of overextrusion and decrease of layer thickness did not increase the density of these materials any more, but resulted in the identical print densities.

\section{Ray Attenuation and Energy Dependence}

Figures 3, 4 show Hounsfield values and their energy dependence for FMM printing materials. In the further discussion, absolute values of $\mathrm{HU}$ are stated at $120 \mathrm{kVp}$, because this tube voltage setting represents the most often used tube potential in CT scanning. The actual exact $\mathrm{HU}$ values measured are available in Table 4

Figure 3 presents the results for nominally unfilled materials (i.e., printing filaments where no filler material such as wood, carbon fiber or mineral powder is specified). In Figure 4 high attenuation filaments potentially mimicking bone tissues, and filled filaments (with declared fillings) are shown.

Natural color and pigmented PLA from two different vendors exhibits similar, but not identical $\mathrm{HU}$ values (difference $8 \mathrm{HU}$ at $120 \mathrm{kVp}$, maximum difference $16 \mathrm{HU}$ at $70 \mathrm{kV}$; Figure 3A). The attenuation of the transparent PLA1 is slightly higher than the attenuation of PLA2 containing a silver color pigment. The PLA/ PBAT based biopolymer exhibits a higher attenuation with approximately $350 \mathrm{HU}$ at $120 \mathrm{kVp}$.

PET and modified PET/PETG based printouts exhibit a wide range of $\mathrm{HU}$ from $<140 \mathrm{HU}$ at $120 \mathrm{kVp}$ to $>270 \mathrm{HU}$ (Figure 3B).

ABS, ASA, high impact polystyrene (HIPS), and polypropylene (PP) form the group of polymers with the lowest $\mathrm{x}$-ray attenuation. In this group, only ABS exhibits an $\mathrm{x}$-ray attenuation exceeding the one of water at $120 \mathrm{kVp}$, as it reconstructs with $30 \mathrm{HU}$. At this energy, ASA mimics water almost perfectly (+2 HU). HIPS $(-40)$ is found close to adipose tissue, and PP is found slightly below $-140 \mathrm{HU}$. The PP light filament exhibits lower $\mathrm{HU}$, with the highest value of approximately $-200 \mathrm{HU}$ at $70 \mathrm{kVp}$ and the lowest at the highest energy $(<-260)$. In Figure 3D the remaining printing materials with higher HU values than soft tissues $(>90$ to $140 \mathrm{HU}$ at $120 \mathrm{kVp}$ ) are summarized. 
TABLE 3 | Optimized printing parameters for the production of phantoms with reproducible x-ray attenuation properties resulting in highest achievable material packing/ density.

\begin{tabular}{|c|c|c|c|c|c|c|c|}
\hline Material & $\begin{array}{c}\text { Nozzle/Bed } \\
\text { Temp. }\left[{ }^{\circ} \mathbf{C}\right]\end{array}$ & $\begin{array}{l}\text { Printing speed } \\
{[\mathrm{mm} / \mathrm{s}]}\end{array}$ & $\begin{array}{c}\text { Flow } \\
\text { rate (\%) }\end{array}$ & $\begin{array}{c}\text { Max. } \\
\text { layer } \\
\text { thickness }[\mathrm{mm}]\end{array}$ & $\begin{array}{c}\text { Density of } \\
\text { printed } \\
\text { sample } \\
{\left[\mathrm{g} / \mathrm{cm}^{3}\right]}\end{array}$ & $\begin{array}{l}\text { Filament density } \\
{\left[\mathrm{g} / \mathrm{cm}^{3}\right]}\end{array}$ & $\begin{array}{c}\text { Difference in } \\
\text { density }\end{array}$ \\
\hline
\end{tabular}

High attenuation filaments

\begin{tabular}{|c|c|c|c|c|c|c|c|}
\hline Vinyl & $230 / 80$ & 40 & 100 & 0.15 & 1.35 & 1.35 & $0.00 \%$ \\
\hline PLA/stone & $220 / 60$ & 50 & 105 & 0.06 & 1.64 & 1.70 & $-3.53 \%$ \\
\hline PLA/chalk & $210 / 70$ & 60 & 115 & 0.06 & 1.39 & 1.40 & $-0.71 \%$ \\
\hline
\end{tabular}

Filled filaments with medium and low attenuation

\begin{tabular}{|c|c|c|c|c|c|c|c|}
\hline PLA-PHA/Glow & $210 / 60$ & 50 & 110 & 0.15 & 1.24 & 1.24 & $0.00 \%$ \\
\hline $\begin{array}{l}\text { PLA-PBAT bio } \\
\text { carbon }\end{array}$ & $220 / 80$ & 60 & 115 & 0.06 & 1.35 & 1.15 & $17.39 \%$ \\
\hline PLA/AI & $210 / 60$ & 50 & 102 & 0.06 & 1.27 & $n / a^{a}$ & $\mathrm{n} / \mathrm{a}$ \\
\hline PLA/wood & $200 / 60$ & 70 & 115 & 0.06 & 1.21 & 1.20 & $0.83 \%$ \\
\hline PLA-PHA/Cork & $230 / 60$ & 50 & 100 & 0.15 & 1.21 & 1.18 & $2.54 \%$ \\
\hline $\begin{array}{l}\text { PETG mod./ } \\
\text { Carbon }\end{array}$ & $230 / 60$ & 50 & 110 & 0.15 & 1.19 & 1.19 & $0.00 \%$ \\
\hline
\end{tabular}

PLA and PLA/PBAT based filaments

\begin{tabular}{|c|c|c|c|c|c|c|c|}
\hline PLA & $210 / 60$ & 50 & 110 & 0.15 & 1.24 & 1.24 & $0.00 \%$ \\
\hline PLA 2 & $210 / 60$ & 50 & 105 & 0.15 & 1.23 & 1.24 & $-0.81 \%$ \\
\hline PLA/PBAT bio & $220 / 80$ & 60 & 115 & 0.06 & 1.35 & 1.39 & $-2.88 \%$ \\
\hline
\end{tabular}

PET and PETG based filaments

\begin{tabular}{|c|c|c|c|c|c|c|c|}
\hline PET & $220 / 75$ & 60 & 115 & 0.06 & 1.33 & 1.34 & $-0.75 \%$ \\
\hline \multirow[t]{2}{*}{ PET mod. } & $260 / 70$ & 40 & 105 & 0.06 & 1.26 & 1.27 & $-0.79 \%$ \\
\hline & & & 110 & 0.15 & & & \\
\hline PET mod. 2 & $240 / 60$ & 60 & 115 & 0.10 & 1.19 & 1.20 & $-0.83 \%$ \\
\hline PETG mod. & $220 / 75$ & 60 & 115 & 0.10 & 1.26 & 1.27 & $-0.79 \%$ \\
\hline
\end{tabular}

ABS, ASA and ASA based filaments

\begin{tabular}{|c|c|c|c|c|c|c|c|}
\hline ABS & $250 / 80$ & 60 & 110 & 0.06 & 1.07 & 1.08 & $-0.93 \%$ \\
\hline ASA & $260 / 100$ & 50 & 115 & 0.06 & 1.06 & 1.07 & $-0.93 \%$ \\
\hline ASA mod. & $260 / 100$ & 50 & 100 & 0.15 & 1.13 & 1.11 & $1.80 \%$ \\
\hline
\end{tabular}

PS, PP, PC and Polyamid based filaments

\begin{tabular}{|c|c|c|c|c|c|c|c|}
\hline HIPS & $240 / 100$ & 40 & 110 & 0.10 & 1.02 & 1.04 & $-1.92 \%$ \\
\hline PP & $240 / 100$ & 25 & 100 & 0.15 & 0.88 & 0.89 & $-1.12 \%$ \\
\hline PP light & $240 / 100$ & 25 & 115 & 0.15 & 0.73 & 0.75 & -2.67 \\
\hline PC & $250 / 90^{b}$ & 40 & 100 & 0.06 & 1.20 & $1.19-1.20$ & $0.00 \%$ \\
\hline Nylon & $250 / 60$ & 45 & 110 & 0.15 & 1.12 & 1.14 & $-1.75 \%$ \\
\hline
\end{tabular}

PU based filaments

$\begin{array}{llllll}\text { TPU } & 230 / 70 & 30 & 100 & 0.15 & 1.16 \quad 0.87 \% \quad 1.15 \quad 1\end{array}$

${ }^{a}$ Not specified by manufacturer.

${ }^{b}$ Cooling fan off.

High attenuation filaments serving as potential candidate materials for mimicking hard tissues with different bone mineral content are shown in Figure 4. Besides Vinyl, two different stone/chalk filled PLA based materials exhibited approximately 500 and $1000 \mathrm{HU}$ at $120 \mathrm{kVp}$. Filled FDM printing polymers (Figure 4B) were found at attenuations from slightly above 100 to approximately $350 \mathrm{HU}$ at $120 \mathrm{kVp}$.

Compared to the wide spectrum of $\mathrm{x}$-ray attenuations found in FDM printing materials, the spectrum of available $\mathrm{x}$-ray attenuations is lower in the resin-based printing technologies (Figure 5). Generally, lower $\mathrm{x}$-ray attenuations can be realized with flexible resins as compared to rigid ones. However, there is an overlap. HU values ranged from slightly over 50 to slightly over 170 at $120 \mathrm{kVp}$. In the Vero polyjet resins, the clear resin (142 $\mathrm{HU}$ at $120 \mathrm{kVp})$ exhibits the lowest attenuation, and the white one (176 HU) the highest indicating the addition of white mineral pigment, possibly titanium oxide.

For most printing materials irrespective of the printing technology the Hounsfield value increases with increasing beam hardness corresponding to a lower effective atomic number than water and soft tissues except adipose tissue. 

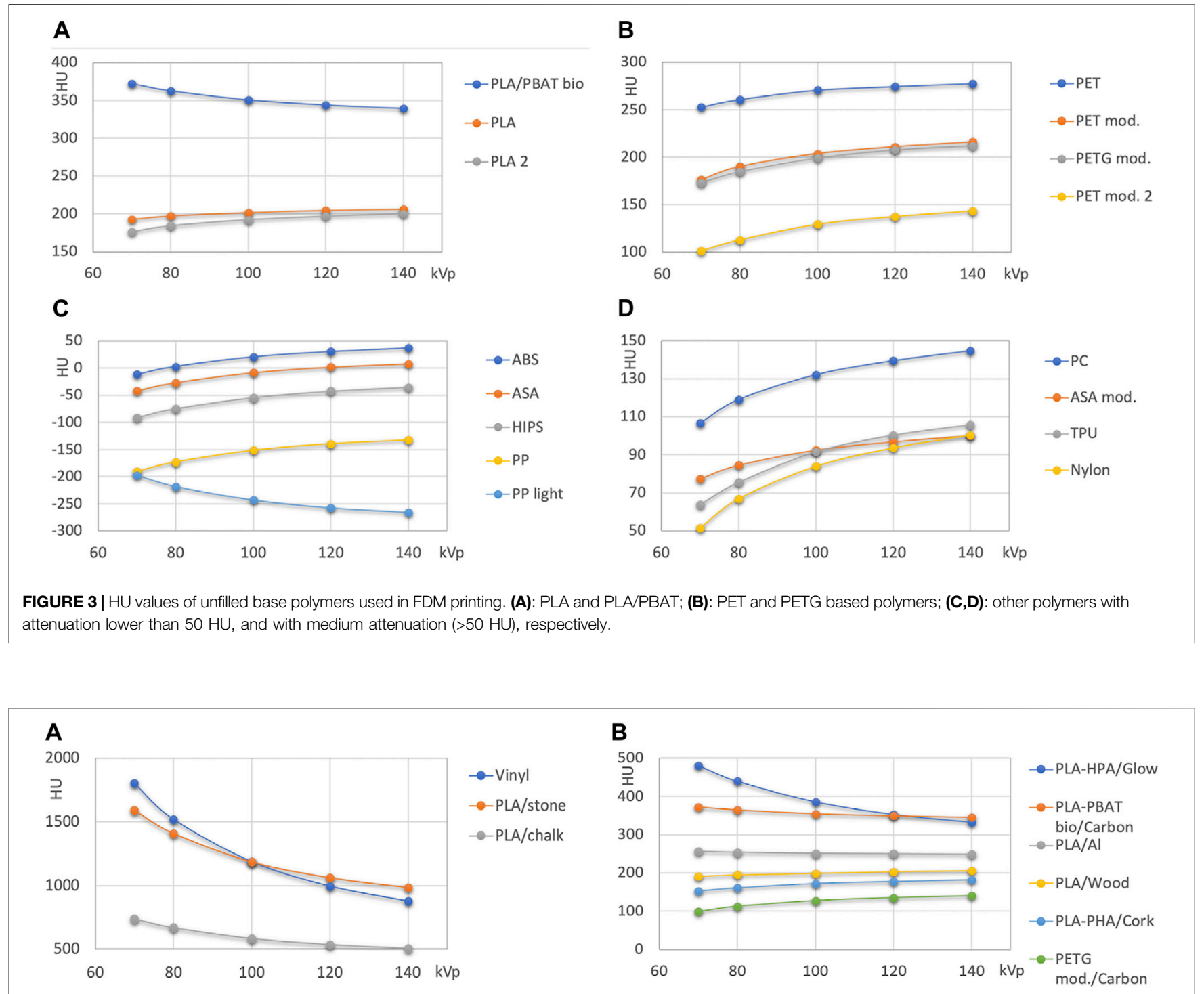

FIGURE 4 | (A): HU values of high attenuation filaments $>500 \mathrm{HU}$. (B): filled polymers with HU values $<500 \mathrm{HU}$.

Regarding unfilled polymers, the only true exemption is Vinyl owned to the higher atomic number of Chlorine. As can be seen in the energy dependence of $\mathrm{HU}$ numbers, the PLA/PBAT biopolymer contains a filling with a higher effective atomic number than water, most likely talcum. In PP light, a filament with a reduced mass density of 0.75 as compared to $0.88-0.92 \mathrm{~g} /$ $\mathrm{cm}^{3}$ for standard polypropylene, embedded air-filled microspheres reduce density. However, these glass microspheres increase the effective atomic number of the material seen in a decrease of HU values with beam hardness.

\section{DISCUSSION}

Available printing materials exhibit a wide range of $\mathrm{x}$-ray attenuations from below -150 to over 1,000 Hounsfield when printed with maximum achievable material density. This range allows the production of radiologic phantoms over a broad gamut of contrasts covering most of the range of attenuations found in patients. However, having established printing parameters resulting in the maximum achievable density, lower attenuations can deliberately and controllably be produced by using infill patterns with reduced filling factor. Many researchers have experimented with varying infill density and patterns to adjust $\mathrm{x}$-ray attenuation properties (Dancewicz et al., 2017) to mimic body tissues. However, this allows a downward adjustment of attenuation, but does not account for the mostly inappropriate energy dependence of most additive manufacturing materials intended to imitate soft tissues like muscle or organ parenchyma. As also stated by Shin et al. (2017) in most polymers tested, HU values increased considerably with increasing tube potential contrary to tissue; in typical soft tissue, Hounsfield numbers 
TABLE 4 | HU values of FDM, polyjet and SLA printing materials in detail.

PLA and PLA based FDM filaments

\begin{tabular}{|c|c|c|c|c|c|c|c|}
\hline$k V p$ & PLA/PBAT bio & PLA & PLA 2 & PET & PET mod. & PETG mod. & PET mod. 2 \\
\hline 70 & 372.1 & 193.0 & 176.7 & 252.8 & 176.4 & 172.7 & 101.0 \\
\hline 80 & 362.7 & 197.5 & 184.6 & 260.6 & 190.2 & 184.7 & 112.7 \\
\hline 100 & 350.7 & 202.0 & 192.5 & 270.5 & 203.7 & 199.0 & 129.3 \\
\hline 120 & 344.1 & 205.0 & 197.2 & 274.4 & 210.9 & 207.6 & 137.5 \\
\hline 140 & 339.6 & 206.6 & 200.4 & 277.6 & 215.9 & 212.1 & 143.3 \\
\hline
\end{tabular}

ABS, ASA, PS and PP filaments: other polymers with $<50 \mathrm{HU}$

\begin{tabular}{|c|c|c|c|c|c|}
\hline & ABS & ASA & HIPS & PP & PP light \\
\hline 70 & -11.7 & -41.4 & -90.9 & -190.4 & -196.9 \\
\hline 80 & 2.9 & -26.5 & -74.3 & -173.0 & -218.0 \\
\hline 100 & 20.6 & -8.2 & -54.0 & -151.6 & -243.2 \\
\hline 120 & 30.4 & 2.1 & -42.2 & -139.6 & -257.8 \\
\hline 140 & 37.0 & 8.2 & -35.0 & -132.7 & -266.1 \\
\hline
\end{tabular}

PC, a modified ASA, TPU and Nylon: other filaments with $>50 \mathrm{HU}$

High attenuation filaments

\begin{tabular}{|c|c|c|c|c|c|c|c|}
\hline & PC & ASA mod. & TPU & Nylon & Vinyl & PLA/stone & PLA/chalk \\
\hline 70 & 106.6 & 77.6 & 64.0 & 51.6 & $1,804.4$ & $1,592.1$ & 738.6 \\
\hline 80 & 119.1 & 84.6 & 75.6 & 67.0 & $1,521.2$ & $1,410.2$ & 669.7 \\
\hline 100 & 132.2 & 92.6 & 91.6 & 84.0 & 1,188.0 & $1,186.7$ & 584.3 \\
\hline 120 & 139.5 & 97.0 & 100.4 & 93.7 & 995.8 & $1,063.1$ & 536.8 \\
\hline 140 & 144.8 & 100.2 & 105.9 & 100.4 & 877.6 & 985.8 & 506.5 \\
\hline
\end{tabular}

Filled filaments with $<500 \mathrm{HU}$

\begin{tabular}{|c|c|c|c|c|c|c|}
\hline & PLA-HPA/GIow & PLA-PBAT bio/Carbon & PLA/Al & PLA/Wood & PLA-PHA/Cork & PETG mod./Carbon \\
\hline 70 & 481.0 & 372.1 & 255.7 & 191.3 & 153.0 & 99.3 \\
\hline 80 & 439.7 & 364.9 & 253.4 & 194.8 & 161.3 & 112.9 \\
\hline 100 & 385.7 & 354.8 & 250.6 & 198.6 & 172.4 & 127.7 \\
\hline 120 & 352.8 & 349.4 & 249.6 & 203.0 & 177.5 & 135.3 \\
\hline 140 & 331.8 & 345.7 & 248.3 & 205.9 & 181.9 & 140.2 \\
\hline
\end{tabular}

Resins for SLA and Polyjet printing: rigid

\begin{tabular}{|c|c|c|c|c|c|c|}
\hline & Vero white & PCre clear & AC translucent & Vero blue & FL clear & Vero clear \\
\hline 70 & 155.9 & 139.2 & 117.3 & 116.5 & 108.6 & 103.8 \\
\hline 80 & 163.6 & 150.4 & 129.8 & 128.3 & 121.1 & 117.3 \\
\hline 100 & 171.3 & 162.7 & 142.9 & 141.5 & 135.3 & 133.3 \\
\hline 120 & 175.7 & 169.7 & 150.7 & 149.0 & 143.5 & 142.1 \\
\hline 140 & 178.3 & 174.3 & 155.2 & 153.5 & 148.5 & 147.2 \\
\hline
\end{tabular}

Resins for SLA and Polyjet printing: flexible

\begin{tabular}{lcccc}
\hline & PCen flex & FL flex & Tango & FL elastic \\
\hline 70 & 118.9 & 65.8 & 56.2 & 15.8 \\
80 & 130.4 & 77.3 & 68.1 & 28.4 \\
100 & 142.6 & 90.7 & 81.4 & 43.2 \\
120 & 149.7 & 98.1 & 89.3 & 52.0 \\
140 & 154.4 & 102.8 & 93.8 & 57.4
\end{tabular}

only vary minimally with $\mathrm{kVp}$ due to their radiological water equivalence, and thus, very similar effective atomic number. The too low effective atomic number $\left(\mathrm{Z}_{\text {eff }}\right)$ of most printing materials with attenuation in the soft tissue range or slightly above results in a $\mathrm{HU}$ value increase with harder beams. This can be compensated by adding a filler with higher atomic number elements. A good example for this is the PLA/Al filament, e.g., exhibiting an almost constant HU value for all spectra measured. However, since unfilled PLA exhibits already a too high density (1.24 $\mathrm{g} / \mathrm{cm}^{3}$ ) and thus linear attenuation coefficient, PLA/Al-despite of the flat energy dependence-cannot serve as a radiological tissue substitute. However, if printed with a lower filling factor the density and attenuation can be reduced in a controllable and reproducible manner. To achieve this goal with a compact phantom material, base polymers with negative HU values like polystyrene or polypropylene (or a 

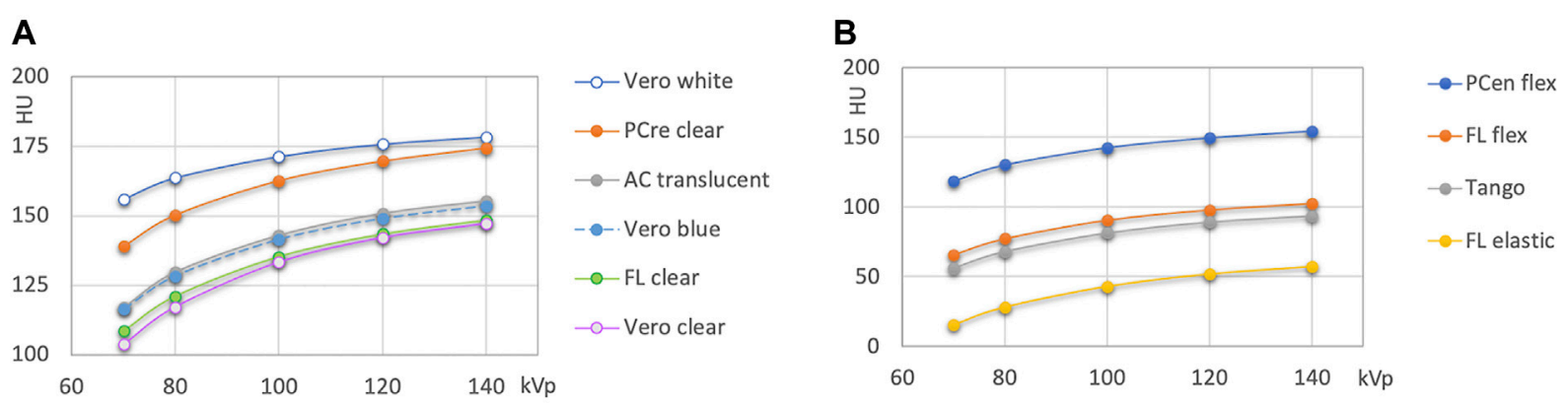

FIGURE 5 | HU values of polyjet and SLA printing resins. (A) rigid, and (B) flexible resins.

mixture of both) would need to be used and higher $Z_{\text {eff }}$ materials added (Homolka et al., 2002). A proven choice would be $\mathrm{MgO}$ and $\mathrm{CaCO}_{3}$. However, such filaments are not available off the shelf.

The achieved densities of the printouts using fused deposition modeling filaments indicated, that most materials can be printed with a density deviation from filament density equal or less than $0.01 \mathrm{~g} / \mathrm{cm}^{3}$ without quality issues like optically detectable artifacts from over-extrusion. However, print settings have to be optimized for each filament individually and carefully since achieved densities may vary in different batches, between printers and between filament manufacturers (Craft et al., 2018). This tedious process includes printing numerous samples with different settings. To illustrate the procedure followed in this work, it is best described by an example. In the case of high impact polystyrene (HIPS) this procedure involved the following steps: Starting from the parameters optimized for general purpose print jobs resulting in a density of $0.99 \mathrm{~g} / \mathrm{cm}^{3}$ compared to the filament density specified as 1.04 by the manufacturer, flow rate was increased from 100 to $110 \%$ resulting in a density increase to $1.01 \mathrm{~g} / \mathrm{cm}^{3}$, since flow rate directly influences mass density, and thus can effectively be used to control the latter (Okkalidis, 2018). In the next step, printing with $115 \%$ flow rater failed because of over-extrusion artifacts. Decreasing layer thickness instead to $0.1 \mathrm{~mm}$ produced a density of $1.02 \mathrm{~g} / \mathrm{cm}^{3}$ still below specifications. However, decreasing layer thickness further to $0.06 \mathrm{~mm}$ did not change density. As a result, $0.1 \mathrm{~mm}$ layer thickness, and $110 \%$ flow rate were defined as the parameters resulting in the highest achievable density as close to raw filament density as possible, even if the specified filament density could not be reproduced in the printout by $0.02 \mathrm{~g} / \mathrm{cm}^{3}$ or $-1.92 \%$.

Also, different infill patterns and wall thickness settings were tested. To achieve a compact printout, lines or circles can be used as infill pattern. However, generally, using lines resulted in a higher density with all other settings identical in the setup used in this work. The same was found for thin versus thicker outer walls. Therefore, for all printed samples the wall thickness was set to its minimum value, and the infill pattern to lines.

Similar polymers from different manufacturers exhibited different properties. This was seen in the case of the most frequently used and supposedly most easy to print thermoplastic polymer, PLA (polylactic acid). The filament supplied from the printer manufacturer (Ultimaker BV, Utrecht, Netherlands) printed with exactly the density specified and typical for PLA $\left(1.24 \mathrm{~g} / \mathrm{cm}^{3}\right)$ using $110 \%$ flowrate. On the contrary, with the other PLA sample tested these $10 \%$ over-extrusion could not be used because of artifacts, and the density specified could not be achieved. This indicates, that filaments from different sources need to be checked on individual basis. However, it needs to be acknowledged that common printing filaments are not specified or optimized for this kind of application.

SLA and polyjet technologies using photocured liquid resins allow for a greater spatial resolution in the prints, but less flexibility in the range of $\mathrm{x}$-ray attenuations. Polyjet technology resins can be mixed during the printing process allowing to print even color gradients. This method allows utilizing the differences in $\mathrm{x}$-ray attenuation of the base materials (the Vero materials in the Stratasys machines, e.g.) to generate phantoms with embedded structures with a selectable radiographic contrast. The highest attenuation was seen in the white material, and a lower attenuation in the transparent. Kiarashi et al. (2015) measured x-ray attenuations of the polyjet printing materials at mammography beam qualities, and found that Vero grey and blue exhibit $\mathrm{x}$-ray attenuation in between white and transparent, and black well below the transparent resin expanding the printable contrast scale.

In the literature various attempts to classify the attenuation of common additive manufacturing materials are available. However, the number of materials tested is more limited than in this work, and some of these need to be read with care. Shin et al. (2017) measured candidate materials for use as analogs for bone, soft tissue, water, and fat from CT scans of filament polymers. The authors scanned a $6 \mathrm{~cm}$ radius phantom containing also resolution patterns and samples printed with different infill percentages. 14 printing materials were evaluated at 80 to $140 \mathrm{kVp}$. However, in the paper no explicit suggestions for candidate materials mimicking bone, soft or adipose tissue, or water, are stated. At $120 \mathrm{kV}$, the $\mathrm{HU}$ value of the materials described range from -55 to $299 \mathrm{HU}$. Using $100 \%$ infill at regular flow rate resulted in potential underfilling ("in several instances, these areas were visibly less dense"), therefore deliberate overfilling was used to result in solid printouts. Due 
to the overfilling, a resolution pattern intended by design to represent 1.6 line pairs per $\mathrm{mm}$ was used to represent the solid material. At $120 \mathrm{kV}$ nylon was measured with $59.1 \mathrm{HU}(93.7 \mathrm{HU}$ in this work) indicating $100 \%$ filling ratio was actually not reached. The same holds true for ABS, where negative HU values at $120 \mathrm{kVp}$ were reported (ABS red: $-49,6$, ABS black: -45.2 ), compared to $+30.4 \mathrm{HU}$ in this work. ABS white is reported with slightly positive $\mathrm{HU}$ (7.3) most likely due to the white pigment contained. HIPS was measured with $-54.7 \mathrm{HU}$ $(120 \mathrm{kVp})$, compared to $-42.2 \mathrm{HU}$ in this work, and PLA with 168.5 (red) and 181.1 (clear) compared to 205.0 here. PET measured corresponded to an industrial PETT filament, and exhibited 165.0 (clear) and 177.9 HU (green) which is also very well below the measurements in this work relating to regular PET. However, the chemical composition of the filament used by Shin et al. is not disclosed by the manufacturer.

In Silvestro et al. (2020) also imaging properties of additive manufacturing materials have been determined for potential use in phantoms, focusing on a wide variety of modalities (ultrasound, MRI and CT). The evaluation in CT was limited to the use of automatic $\mathrm{kV}$ selection by the scanner, not allowing to derive information on energy dependence. In addition, the interpretation of the results is complicated by not reporting the actual $\mathrm{kVp}$ value selected by the scanner. The authors determined the CT numbers of solid ASA and ABS with 16.6 and $57.5 \mathrm{HU}$, respectively, and Tango and VeroClear with 98.1 and $146.6 \mathrm{HU}$. In this work, employing defined and appropriate scan conditions by embedding the samples into a water filled cylinder, plain ASA and ABS (without filling materials and color pigments) were measured with at 2.1 and $30.4 \mathrm{HU}$ at $120 \mathrm{kV}$, respectively, and much lower values at lower $\mathrm{kVp}$, and higher values for harder spectra. Tango and Vero clear were found in this work at 89.3 and $142.1 \mathrm{HU}(120 \mathrm{kV})$. However, the phantom used by Silvestro et al. (2020) consisted of $4.5 \times 2.6 \times 0.65 \mathrm{~cm}$ polymer samples evaluated, embedded into a poured silicone block $75 \mathrm{~mm}$ high. The form, size, and material of the embedding will not allow the CT's built-in beam hardening correction to derive correct $\mathrm{HU}$ numbers of the samples. Especially silicone embedding should be avoided because of the too high atomic number of silicon resulting in issues with the beam hardening correction aiming at avoiding cupping and reverse cupping in patients and radiographically water equivalent materials only. HU measured this way will most likely not reflect those measured in more appropriate scan condition as used in this work. This discrepancy outlines the importance of using appropriate scan conditions including reporting technical scan parameters like $\mathrm{kVp}$ if correct and reproducible $\mathrm{HU}$ numbers shall be reported.

Bibb et al. (2011) measured CT values of 14 solid additive manufacturing material samples of $40 \times 20 \times 10 \mathrm{~mm}$ embedded in a low density foam block ("free in air"), and attached to a head phantom at $120 \mathrm{kV}$ using a bone reconstruction kernel. However, no care was taken to ensure a complete filling of the FDM printed samples, and the CT image provided in the figure clearly exhibits a linear inner structure of the samples, indicating inhomogeneities due to underfilling. The authors state that "some samples are not homogeneous and their density varies considerably across their sections", indicating that CT numbers were strongly influenced by the printing process and might not be attributed to the material attenuation properties only, as desired. However, this example clearly shows the necessity to optimize the printing parameters (mostly, layer height and deliberate overextrusion in FDM) very carefully for every material and individual printer before printing phantoms to avoid underfilling or inhomogeneous densities. Also, scanning conditions influence the CT numbers mostly due to the effect of the beam hardening correction. In Bibb et al. (2011) up to 128 HU difference (average $28 \mathrm{HU}$ ) is reported for the same sample scanned either on the head phantom, or in the foam block. This difference, again, emphasizes the importance of well-defined scanning conditions, as is best demonstrated by CT quality control or electron density calibration phantoms used in radiotherapy planning (Constantinou et al., 1992; Nakao et al., 2020), where the calibration cylinders are embedded into a water equivalent material in a cylindrical phantom of appropriate diameter.

Other studies aimed at suggesting commonly available additive manufacturing materials explicitly for mimicking tissue in radiographic phantoms. Kairn et al. (2020) concluded, that PLA filaments with a Calcium based filling, like StoneFil (Formfutura BV, Nijmegen, Netherlands) represent an appropriate surrogate for hard bone at $\mathrm{kV}$ and MV energies which is in accordance with this study. Ivanov et al. (2018) studied the suitability of common low density 3D printing materials for the fabrication of breast phantoms. Their conclusion was, that most SLA resins, as well as Nylon and PET-G exhibit attenuation characteristics close to glandular tissue, while ABS more closely mimics adipose tissue at mammographic energies, and thus, represent possible material candidates for $3 \mathrm{D}$ printed phantoms.

Printing different materials in one phantom is limited to few printing technologies, like, e.g., polyjet printing. Some FDM printers using a dual head extrusion system are limited to printing two materials simultaneously. However, phantoms parts made of different materials, and even made with different technologies possibly combining the high spatial resolution from photocured resins with the wide range of $x$-ray attenuation found in filled FDM filaments, can be printed separately and assembled. Another possibility is using castable materials filled in voids of the printed base phantoms, as has been done in (Hatamikia et al., 2020) e.g., where soft tissue was printed and bone mimicking material then cast in the voids spared in the printing process using bone mineral and polypropylene powder mixed into epoxy resin. In (Cockmartin et al., 2017) anthropomorphic breast lesions were printed, and assembled into a phantom filled with PMMA speres and water simulating the anatomical background.

\section{CONCLUSION}

When determining the radiation attenuation properties of additive manufacturing materials (and, materials in general), printing parameters and scan conditions need to be selected 
carefully and appropriately, what has not always been the case in published studies. For FDM printing, it is indispensable to assure the maximum achievable filling ratio is achieved prior to printing the test samples.

The energy dependence of low and medium attenuation additive manufacturing polymers is mostly unfavorable due to the low effective atomic number. While the linear attenuation coefficient, of course, decreases with photon energy, it decreases less strongly than in water, resulting in a relative increase of $\mathrm{HU}$ values with harder beams. This makes it difficult to print materials with linear attenuation coefficients similar to muscle tissue or organ parenchyma over an extended energy range. However, spectrum and energy independent attenuation relative to water can be realized by adding filler materials with a higher atomic number. However, in readily available industrial and consumer grade printing materials the choice is limited. For high attenuation tissues like bone tissues, calcium filled polymers provide both, favorable energy dependence, and attenuation values.

The measurements performed in this study can be used to design phantoms with a wide range of $x$-ray contrasts. Using the information on the energy dependence from 70 to $140 \mathrm{kVp}$, materials with similar energy dependence can be selected to allow (almost) energy independent contrasts. If the energy dependence of the intended phantom contrast is known, the most appropriate materials may be selected and the absolute value of the linear attenuation adjusted downwards by using

\section{REFERENCES}

\#3DBenchy (2021). Creative Tools, Halmstad, Sweden. [Online]. Available at: http://www.3dbenchy.com/ (Accessed Aug. 24, 2021).

Bibb, R., Thompson, D., and Winder, J. (2011). Computed Tomography Characterisation of Additive Manufacturing Materials. Med. Eng. Phys. 33, 590-596. doi:10.1016/j.medengphy.2010.12.015

Carton, A.-K., Bakic, P., Ullberg, C., Derand, H., and Maidment, A. D. A. (2011). Development of a Physical 3D Anthropomorphic Breast Phantom. Med. Phys. 38, 891-896. doi:10.1118/1.3533896

Cockmartin, L., Marshall, N. W., Zhang, G., Lemmens, K., Shaheen, E., Van Ongeval, C., et al. (2017). Design and Application of a Structured Phantom for Detection Performance Comparison between Breast Tomosynthesis and Digital Mammography. Phys. Med. Biol. 62, 758-780. doi:10.1088/ 1361-6560/aa5407

Constantinou, C., Harrington, J. C., and Dewerd, L. A. (1992). An Electron Density Calibration Phantom for CT-based Treatment Planning Computers. Med. Phys. 19, 325-327. doi:10.1118/1.596862

Craft, D. F., Kry, S. F., Balter, P., Salehpour, M., Woodward, W., and Howell, R. M. (2018). Material Matters: Analysis of Density Uncertainty in 3D Printing and its Consequences for Radiation Oncology. Med. Phys. 45, 1614-1621. doi:10.1002/ mp.12839

Dancewicz, O. L., Sylvander, S. R., Markwell, T. S., Crowe, S. B., and Trapp, J. V. (2017). Radiological Properties of 3D Printed Materials in Kilovoltage and Megavoltage Photon Beams. Physica Med. 38, 111-118. doi:10.1016/ j.ejmp.2017.05.051

Filippou, V., and Tsoumpas, C. (2018). Recent Advances on the Development of Phantoms Using 3D Printing for Imaging with CT, MRI, PET, SPECT, and Ultrasound. Med. Phys. 45, e740-e760. doi:10.1002/mp.13058

Hatamikia, S., Oberoi, G., Unger, E., Kronreif, G., Kettenbach, J., Buschmann, M., et al. (2020). Additively Manufactured Patient-Specific Anthropomorphic Thorax Phantom with Realistic Radiation Attenuation Properties. Front. Bioeng. Biotechnol. 8, 385. doi:10.3389/fbioe.2020.00385 under extrusion and adjusted infill ratios. The data provided on the energy dependence can also be used to correct contrast or contrast to noise ratios measured in phantoms made from a wide selection of additive manufacturing materials to simulate anatomical contrasts from tissues with known, but different energy dependence. These include contrasts between different soft tissues (organ parenchyma, muscle, adipose tissue to name some of the most important), lung tissue, and bone of various densities.

\section{DATA AVAILABILITY STATEMENT}

The original contributions presented in the study are included in the article/Supplementary Material, further inquiries can be directed to the corresponding author.

\section{AUTHOR CONTRIBUTIONS}

Guarantor for the integrity of the entire study: PH; Study concepts and design: $\mathrm{PH}, \mathrm{XM}$; 3D printing: $\mathrm{XM}, \mathrm{EU}$; Phantom design and fabrication: XM, CT data generation and evaluation: $\mathrm{XM}, \mathrm{PH}, \mathrm{MB}$; Manuscript preparation: $\mathrm{PH}$; Approval of the final version of the manuscript submitted: all authors.

Homolka, P., and Nowotny, R. (2002). Production of Phantom Materials Using Polymer Powder Sintering under Vacuum. Phys. Med. Biol. 47, N47-N52. doi:10.1088/0031-9155/47/3/401

Homolka, P., Gahleitner, A., Prokop, M., and Nowotny, R. (2002). Optimization of the Composition of Phantom Materials for Computed Tomography. Phys. Med. Biol. 47, 2907-2916. doi:10.1088/0031-9155/47/16/306

Homolka, P., Figl, M., Wartak, A., Glanzer, M., Dünkelmeyer, M., Hojreh, A., et al. (2017). Design of a Head Phantom Produced on a 3D Rapid Prototyping Printer and Comparison with a RANDO and 3M Lucite Head Phantom in Eye Dosimetry Applications. Phys. Med. Biol. 62, 3158-3174. doi:10.1088/1361$6560 / \mathrm{aa} 602 \mathrm{c}$

IAEA (2007). “TRS 457. Dosimetry in Diagnostic Radiology: An International Code of Practice," in Technical Reports Series (Vienna: IAEA).

Irnstorfer, N., Unger, E., Hojreh, A., and Homolka, P. (2019). An Anthropomorphic Phantom Representing a Prematurely Born Neonate for Digital X-ray Imaging Using 3D Printing: Proof of Concept and Comparison of Image Quality from Different Systems. Sci. Rep. 9, 14357. doi:10.1038/s41598-019-50925-3

Ivanov, D., Bliznakova, K., Buliev, I., Popov, P., Mettivier, G., Russo, P., et al. (2018). Suitability of Low Density Materials for 3D Printing of Physical Breast Phantoms. Phys. Med. Biol. 63, 175020. doi:10.1088/1361-6560/ aad315

Kairn, T., Zahrani, M., Cassim, N., Livingstone, A. G., Charles, P. H., and Crowe, S. B. (2020). Quasi-simultaneous 3D Printing of Muscle-, Lung- and BoneEquivalent media: a Proof-Of-Concept Study. Phys. Eng. Sci. Med. 43, 701-710. doi:10.1007/s13246-020-00864-5

Kalender, W. A., Suess, C., and Faust, U. (1988). Polyethylene-based Water- and Bone-equivalent Materials for Calibration Phantoms in Quantitative Computed Tomography - Ein Kalibrierphantom für die quantitative Computertomographie aus wasser- und knochenäquivalentem Material. Biomed. Tech. (Berl.) 33, 73-76. doi:10.1515/bmte.1988.33.4.73

Kiarashi, N., Nolte, A. C., Sturgeon, G. M., Segars, W. P., Ghate, S. V., Nolte, L. W. et al. (2015). Development of Realistic Physical Breast Phantoms Matched to Virtual Breast Phantoms Based on Human Subject Data. Med. Phys. 42, 4116-4126. doi:10.1118/1.4919771 
Nakao, M., Ozawa, S., Miura, H., Yamada, K., Habara, K., Hayata, M., et al. (2020). Development of a CT Number Calibration Audit Phantom in Photon Radiation Therapy: A Pilot Study. Med. Phys. 47, 1509-1522. doi:10.1002/ mp.14077

Okkalidis, N. (2018). A Novel 3D Printing Method for Accurate Anatomy Replication in Patient-specific Phantoms. Med. Phys. 45, 4600-4606. doi:10.1002/mp.13154

Schopphoven, S., Cavael, P., Bock, K., Fiebich, M., and Mäder, U. (2019). Breast Phantoms for 2D Digital Mammography with Realistic Anatomical Structures and Attenuation Characteristics Based on Clinical Images Using 3D Printing. Phys. Med. Biol. 64, 215005. doi:10.1088/1361-6560/ab3f6a

Shin, J., Sandhu, R. S., and Shih, G. (2017). Imaging Properties of 3D Printed Materials: Multi-Energy CT of Filament Polymers. J. Digit Imaging 30, 572-575. doi:10.1007/s10278-017-9954-9

Silvestro, E., Betts, K. N., Francavilla, M. L., Andronikou, S., and Sze, R. W. (2020). Imaging Properties of Additive Manufactured (3D Printed) Materials for Potential Use for Phantom Models. J. Digit Imaging 33, 456-464. doi:10.1007/s10278-019-00257-5

Smet, M. H., Breysem, L., Mussen, E., Bosmans, H., Marshall, N. W., and Cockmartin, L. (2018). Visual Grading Analysis of Digital Neonatal Chest Phantom X-ray Images: Impact of Detector Type, Dose and Image Processing on Image Quality. Eur. Radiol. 28, 2951-2959. doi:10.1007/s00330-017-5301-2

Solomon, J., Bochud, F., and Samei, E. (2014). "Design of Anthropomorphic Textured Phantoms for CT Performance Evaluation," in Medical Imaging 2014: Physics of Medical Imaging. San Diego, CA: SPIE. doi:10.1117/ 12.2043555

Sousa, M. a. Z., Matheus, B. R. N., and Schiabel, H. (2018). Development of a Structured Breast Phantom for Evaluating CADe/Dx Schemes Applied on 2D Mammography. Biomed. Phys. Eng. Express 4, 045018. doi:10.1088/2057-1976/aac2f2
Spelic, D. C., Kaczmarek, R. V., and Suleiman, O. H. (2004). Nationwide Evaluation of X-ray Trends Survey of Abdomen and Lumbosacral Spine Radiography. Radiology 232, 115-125. doi:10.1148/radiol.2321020397

Suleiman, O. H., Stern, S. H., and Spelic, D. C. (1999). Patient Dosimetry Activities in the United States: the Nationwide Evaluation of X-ray Trends (NEXT) and Tissue Dose Handbooks. Appl. Radiat. Isot. 50, 247-259. doi:10.1016/s09698043(98)00073-6

White, D. R., Martin, R. J., and Darlison, R. (1977). Epoxy Resin Based Tissue Substitutes. Br. J. Radiol. 50, 814-821. doi:10.1259/0007-1285-50599-814

Conflict of Interest: The authors declare that the research was conducted in the absence of any commercial or financial relationships that could be construed as a potential conflict of interest.

Publisher's Note: All claims expressed in this article are solely those of the authors and do not necessarily represent those of their affiliated organizations, or those of the publisher, the editors and the reviewers. Any product that may be evaluated in this article, or claim that may be made by its manufacturer, is not guaranteed or endorsed by the publisher.

Copyright (C) $2021 \mathrm{Ma}$, Buschmann, Unger and Homolka. This is an open-access article distributed under the terms of the Creative Commons Attribution License (CC $B Y)$. The use, distribution or reproduction in other forums is permitted, provided the original author(s) and the copyright owner(s) are credited and that the original publication in this journal is cited, in accordance with accepted academic practice. No use, distribution or reproduction is permitted which does not comply with these terms. 